\title{
Percepção dos Coordenadores das Equipes de Saúde da Família de Gurupi/TO sobre a Implementação da Política Nacional de Atenção Básica
}

\section{Perception of the Coordinators of Gurupi/TO Family Health Teams on the Implementation of the National Policy of Basic Attention}

Jeann Bruno Ferreira da Silva1, Marta Azevedo dos Santos², Aline da Silveira Gonçalves Fabris ${ }^{3}$, Marcia Andrea Marroni4, Leila Rute Oliveira Gurgel do Amaral $^{5}$, Pammalla Ribeiro da Conceição ${ }^{6}$

\section{RESUMO}

A Atenção Básica é caracterizada como a principal porta de entrada do Sistema Único de Saúde, na qual devem estar centradas as ações de promoção da saúde. Para o seu pleno desenvolvimento é necessário que os atores envolvidos neste processo compreendam a importância de se pautarem nos princípios da Política Nacional de Atenção Básica (PNAB). O objetivo desta pesquisa consistiu em compreender a percepção dos coordenadores das Equipes de Saúde da Família (ESF) sobre a implementação da PNAB nas Unidades Básicas de Saúde (UBS) do município de Gurupi-TO. Participaram da pesquisa 11 (onze) enfermeiros coordenadores das ESF, um de cada UBS. Tratou-se de uma pesquisa descritivo-e exploratória, de abordagem qualitativa. Para análise dos dados utilizou-se o método de análise de conteúdo de Bardin (2009). No município de Gurupi, o processo de implementação da PNAB ocorre de maneira vertical, através de reuniões mensais com gestores da Secretaria Municipal de Saúde, no entanto, por mais que os sujeitos participantes desta pesquisa reconheçam que os princípios e diretrizes da referida política pública de saúde são importantes para o pleno desenvolvimento da Atenção Básica, os mesmos afirmaram que, devido à sobrecarga de tarefas e atribuições e ao déficit de recursos humanos, a implementação da PNAB não ocorre integralmente.

Palavras-Chave: Atenção Básica. Política Pública. Promoção da saúde.

\section{ABSTRACT}

Basic attention is characterized as the main gateway of the single healthcare system, in which health promotion actions should be centered. For its full development it is necessary that the actors involved in this process understand the importance of being guided by the principles of the National Basic Attention Policy (PNAB). The objective of this survey consisted of understanding the perception of the coordinators of the Family Health Teams (ESF) on the implementation of the PNAB in the basic health units (UBS) of the municipality of Gurupi-TO. Participated in the survey 11 (eleven) Nurses coordinators of the ESF, one of each UBS. It was a descriptive and exploratory survey of qualitative approach. For analysis of the data, the method of analysis of Bardin's Content (2009) was used. In the municipality of Gurupi, the process of implementing the PNAB occurs in a vertical manner, through monthly meetings with managers of the municipal Health Secretariat, however, for more than the participants in this research acknowledge that the principles and guidelines of the public health policy are important for the full development of basic attention, they have stated that, owing to the burden of tasks and assignments and the human resources deficit, the implementation of PNAB does not occur fully.

Keywords: Basic Attention. Public policy. Health promotion.

Mestre em Ciências da Saúde pela UFT. Graduado em Psicologia. $\quad$ Professor Especialista do Centro Universitário UnirG, Gurupi-TO.

E-mail: jbpsicologia@live.com

Doutora em Psicologia. Graduada em Psicologia. Professora e Orientadora do Mestrado Profissional em Ciências da Saúde na UFT. Colaboradora Técnica do Ministério da Saúde.

${ }^{3}$ Mestre em Ciências da Saúde pela UFT. Graduada em Psicologia - Unoesc-SC.

${ }^{4}$ Doutora em Saúde do Adulto pela Escola de Enfermagem da USP. Graduada em Enfermagem e Obstetrícia. Professora titular no Centro Universitário UnirG, Gurupi-TO.

${ }^{5}$ Pós-doutora em Psicologia da Educação pela UNESP. Doutora em Ciências pela USP. Graduada em Psicologia. Professora adjunta do colegiado de Medicina e do Mestrado Profissional em Ciências da Saúde na UFT.

${ }^{6}$ Pós-graduada Implantodontia pela Faculdade de Conchas - FACON. Graduada em Odontologia - UnirG/TO. 


\section{INTRODUÇÃO}

Desde a criação do Sistema Único de Saúde (SUS) no Brasil, é possível reconhecer e identificar os visíveis avanços na ampliação e acesso que a população tem possuído tanto nas ações de saúde da Atenção Básica $(A B)$ quanto na rede de alta complexidade. Considera-se então que estes sejam reflexos de planejamento, organização, arranjos e de investimentos nas equipes de saúde que compõem o sistema. Assim, são garantidos os princípios da universalidade, da equidade, da integralidade, da descentralização, da regionalização, da hierarquização e da participação social. Sobre a garantia destes princípios, as políticas públicas de saúde têm este propósito.

Entende-se por política pública um conjunto de dispositivos, medidas e procedimentos que expressam a orientação política do Estado e regulam as atividades governamentais relacionadas às tarefas de interesse público, atuando e influindo sobre as realidades econômicas sociais e ambientais, traduzindo a ideia de que um Estado existe em função de um interesse maior, da sociedade, e será expressão daquilo que a sociedade almeja e necessita (GIOVANELLA, 2009).

No caso da Política Nacional de Atenção Básica (PNAB), por se tratar de uma política pública de saúde, a mesma garante o pleno acesso da população aos serviços de saúde e aborda questões desde a participação da comunidade na gestão da $A B$ ao estabelecimento de mecanismos que assegurem acessibilidade e acolhimento no processo de trabalho das equipes de saúde.

Amparado pelo Art. 198 da Constituição Federal, bem como pela sua regulamentação através da Lei ํㅜ 8.080/90, o SUS baseou-se na formulação de um modelo de saúde voltado para as demandas e necessidades da população, consolidando a saúde como um direito a partir do compromisso do Estado com o bem-estar social.

A PNAB firma o compromisso de melhoria das condições da assistência à saúde da população, constituindo a $A B$ como a principal porta de entrada do SUS. A nova PNAB foi aprovada pela Portaria 2.488 , de 21 de outubro de 2011 revoga a anterior, aprovada pela Portaria no 648/GM de 28 de março de 2006, e que estabelece a revisão de diretrizes e normas para organização da $A B$, contudo, há de se considerar que ela é resultado da trajetória histórica e da experiência acumulada de lutas de movimentos sociais, usuários, trabalhadores e gestores das três esferas de governo.

Com a edição da PNAB o governo federal estabeleceu diretrizes para o fortalecimento da APS no sistema de saúde brasileiro. A Estratégia da Saúde da Família (ESF) ganhou 
importância como política governamental e passou a ser prioritária para a organização do sistema público de serviços de saúde.

Dentre os fundamentos e diretrizes da PNAB, destacam-se o estímulo e participação dos usuários como maneira de ampliar sua autonomia e capacidade na construção do cuidado à saúde das pessoas e coletividades do território, no enfrentamento dos determinantes e condicionantes de saúde, na organização e orientação dos serviços a partir de lógicas mais centradas no usuário e no exercício do controle social (BRASIL, 2012).

Outro aspecto a ser observado refere-se aos termos Atenção Básica e Atenção Primária à Saúde, propostos pela PNAB como termos equivalentes, com significações de mesmo cunho, pois nesse processo histórico, a $A B$ foi gradualmente se fortalecendo e se tornando o ponto de partida para a estruturação dos sistemas locais de saúde.

A relevância da PNAB não se reduz somente à sua articulação com uma gama de atividades voltadas ao cuidado e à prevenção, pois a mesma também assume o papel de capilarizar o SUS por todo o país, formalizando e instrumentalizando a organização dos modelos locais de saúde.

A PNAB também enfatiza que o processo de trabalho das equipes de Atenção Básica (eAB) centra-se em atender toda a população adstrita no território de abrangência das UBS. Assim, as equipes devem programar e implementar as atividades de atenção à saúde de acordo com as necessidades da população, priorizando intervenções clínicas e sanitárias nos problemas de saúde segundo critérios de frequência, risco, vulnerabilidade e resiliência (BRASIL, 2012). Outra prática das $e A B$ prevista na $P N A B$, refere-se à utilização e articulação de diferentes tecnologias de cuidado individual e coletivo para com os usuários. Para tal fim, a equipe utiliza como meio uma clínica ampliada capaz de construir vínculos positivos com os usuários do sistema. A clínica ampliada é a diretriz de atuação dos profissionais da saúde que prevê, de acordo com a necessidade dos usuários, a articulação entre os serviços de saúde e outros setores e políticas públicas. Este conceito pauta-se na articulação e diálogo de diferentes saberes para compreensão dos processos de saúde e adoecimento (SUNDFELD, 2010). Um dos conceitos discutidos na PNAB refere-se à organização de Redes de Atenção à Saúde (RAS) como estratégias para estabelecimento do cuidado integral e direcionado às necessidades de saúde da população. Para que o funcionamento das RAS seja executado de maneira plena é necessário que a AB cumpra sua função de coordenação do cuidado, acompanhamento e gestão de Projetos Terapêuticos Singulares (BRASIL, 2012). 
O Projeto Terapêutico Singular é produto da discussão coletiva dos profissionais de saúde da $A B$ e do compartilhamento com o usuário, pois as condutas terapêuticas e as metas são negociadas. Baseia-se na singularidade do usuário, na receptividade das diferenças que transcendem os diagnósticos e sua inclinação para igualar os sujeitos (BRASIL, 2009). Assim, as equipes cumprem o que é proposto na PNAB.

Dentro desse contexto, a pesquisa objetivou responder a seguinte questão norteadora: Como ocorre o processo de implementação e organização da PNAB nas UBS do município de Gurupi-TO. Para que isto fosse possível, estabeleceu-se como crivo a percepção dos coordenadores das Equipes de Saúde da Família sobre o referido processo.

\section{MATERIAIS E METODOS}

Trata-se de um estudo descritivo-exploratório, com abordagem qualitativa. A abordagem qualitativa justifica-se pelo fato da mesma permitir o conhecimento das ações realizadas pelos coordenadores das ESF, portanto, produziu conhecimentos para aplicação prática dirigida à solução de problemas específicos.

A pesquisa qualitativa foi empregada como uma possibilidade de aprofundar a compreensão do fenômeno em investigação, com ênfase nos processos vivenciados e nos significados atribuídos pelos sujeitos pesquisados em questão (MINAYO, 2007).

O caráter descritivo tem por finalidade descrever as características de uma determinada população ou fenômeno que tem como uma de suas peculiaridades a utilização de técnicas padronizadas de coleta de dados, tais como o questionário e a observação sistemática. Já o caráter exploratório tem por finalidade proporcionar maior familiaridade com o problema, com vistas a desenvolver, esclarecer e modificar conceitos e ideias para estudos posteriores (GIL; DESLAURIERS; KÉRISIT, 2008).

A pesquisa ocorreu nas 11 (onze) UBS do município de Gurupi, Estado do Tocantins, no período de outubro a dezembro do ano de 2016. Vale ressaltar que estas 11 (onze) UBS são coordenadas por enfermeiros das ESF. Também foi realizado contato com a Coordenação de Atenção Básica do município para complementar informações acerca do processo de implementação do fluxograma da PNAB.

Os critérios de inclusão utilizados foram: estar na coordenação da ESF no período mínimo de 1 (um) ano, aceitar participar da pesquisa e assinar o Termo de Consentimento Livre e Esclarecido - TCLE. Foram excluídos do estudo os colaboradores que não ocuparam e exerciam a função de coordenador ou responsável, bem como àqueles que estiveram interinamente na função em substituição a outrem por motivo de férias, licenças 
e/ou afastamentos, os que não aceitaram participar do estudo estando em desacordo com o TCLE. De uma população de 22 (vinte e dois) enfermeiros, aplicando-se os critérios de inclusão e exclusão anteriormente mencionados, fizeram parte deste estudo 11 (onze) coordenadores de ESF.

O estudo pautou-se conforme previsto na resolução 466/2012 do Conselho Nacional de Saúde, foi submetido ao Comitê de Ética em Pesquisa com Seres Humanos da Universidade Federal do Tocantins sob CAAE: 59451616.5.0000.5519 e aprovado conforme parecer $n^{\circ} 1.780 .003$. Antes do início da coleta de dados foi lido o Termo de Consentimento Livre e Esclarecido para o participante, o qual consentia ou não em participar do estudo, logo após a coleta de dados foi realizada a entrevista por meio de roteiro semiestruturado.

Todas as entrevistas foram gravadas em aparelho MP4 e logo após, no mesmo dia, ocorreu o processo de transcrição de modo a evitar a perda dos detalhes das falas dos entrevistados, assegurando sua respectiva fidedignidade. Como forma de compreender o objeto de estudo em suas múltiplas dimensões, utilizou-se um diário de campo, com o propósito de observar e registrar a dinâmica do local, ações realizadas com os entrevistados, percepções e sentimentos do pesquisador acerca de tal contexto.

O diário de campo é fundamental para registrar as percepções diárias do pesquisador, seja de forma escrita, seja de forma gravada. Devem-se registrar conversas informais, observações de comportamento, falas e impressões pessoais (que normalmente vão se modificando com o tempo) sobre as categorias ou pontos a serem investigados (GUERRA, 2014).

Quanto à análise e interpretação das entrevistas, utilizou-se a técnica de análise de conteúdo de Bardin (2009), à qual consiste num conjunto de procedimentos e técnicas que visam extrair sentido dos textos por meio de unidades de análises que podem ser palavraschaves, termos específicos, categorias e/ou temas, de modo a identificar a frequência com que aparecem no texto, possibilitando fazer inferências replicáveis e válidas dos dados. A mesma é constituída pelas seguintes etapas: pré-análise, exploração do material e tratamento dos resultados, inferência e interpretação (BARDIN, 2009).

$\mathrm{Na}$ fase inicial (pré-análise), realizou-se a etapa de leitura flutuante, a fim de estabelecer contato com o material coletado. Após esta etapa, foi realizada a escolha dos documentos a serem analisados, neste caso, as entrevistas, que foram devidamente transcritas. 
$\mathrm{Na}$ etapa final preparou-se o material através de recortes e edição das entrevistas transcritas, possibilitando assim a identificação de indicadores. Para que isso fosse possível, foi necessário organizar as entrevistas em colunas, para anotar e marcar semelhanças e contrastes destacando-os em diferentes cores.

Somente após os procedimentos anteriores, foram estabelecidos os graus de similaridade entre as informações contidas nas diversas entrevistas, sendo possível o agrupamento de cada trecho, conforme núcleos de pensamento contido em cada um deles. Enfim, a codificação do material incluiu a escolha de unidades de registro e a seleção de regras de contagem.

\section{RESULTADOS E DISCUSSÖES}

Após a leitura das entrevistas foram elaboradas as Unidades de Registro e, a partir destas, foram determinadas as Unidades de Contexto, buscando-se segmentos da mensagem que auxiliaram para sua compreensão exata, ou seja, para codificá-las. Subsequentemente às etapas anteriores, realizou-se o processo de categorização, tornando-se necessário realizar o tratamento e interpretação dos resultados pelo aspecto léxico, ou seja, de acordo com o seu sentido, conforme apresentado na Tabela 1.

A categoria final de análise - Implementação do fluxograma de PNAB - apresenta a percepção e conhecimento dos profissionais acerca da PNAB, bem como descreve a organização e orientações da gestão sobre a mesma.

Desde o surgimento do SUS e seu respectivo processo de descentralização políticoadministrativa, a implementação da $A B$ tornou-se responsabilidade, principalmente, dos gestores municipais. Tal processo de descentralização do sistema prevê a organização local com estruturas político-administrativas que sejam responsáveis pelas políticas públicas (SOUZA; MACHADO; NORONHA, 2015). No município de Gurupi este local é contemplado na SEMUS, onde possui dois departamentos específicos de políticas públicas em saúde, são eles a Direção de Atenção Básica e a Coordenação de Atenção Básica, ambos são responsáveis por implementá-las, fiscalizá-las e avaliá-las.

Em consonância ao exposto, para o pleno desenvolvimento da $A B$ nos territórios, torna-se necessário que toda a equipe inserida em tal processo obtenha conhecimento mínimo das diretrizes da PNAB. 
Tabela 1. Unidades de Registro, Unidades de Contexto e Categoria final de Análise identificadas nas entrevistas com os coordenadores das ESF do município de Gurupi, Tocantins, Brasil.

\begin{tabular}{|c|c|c|}
\hline UNIDADES DE REGISTRO & $\begin{array}{l}\text { UNIDADES DE } \\
\text { CONTEXTO }\end{array}$ & $\begin{array}{l}\text { CATEGORIA FINAL } \\
\text { DE ANÁLISE }\end{array}$ \\
\hline Conhecimento parcial sobre a PNAB & \multirow{3}{*}{$\begin{array}{c}\text { Percepção } \\
\text { sobre a PNAB }\end{array}$} & \multirow{17}{*}{$\begin{array}{c}\text { IMPLEMENTAÇÃO } \\
\text { DO FLUXOGRAMA } \\
\text { DA PNAB }\end{array}$} \\
\hline Convicção sobre o conhecimento da PNAB & & \\
\hline Não conhecimento da PNAB & & \\
\hline Interesse em conhecer mais sobre a PNAB & \multirow{4}{*}{$\begin{array}{c}\text { A PNAB como } \\
\text { desafio }\end{array}$} & \\
\hline A importância da PNAB & & \\
\hline Divulgação da PNAB por parte da gestão & & \\
\hline Ausência de um exemplar da PNAB na UBS & & \\
\hline Reuniões com a Secretaria de Saúde & \multirow{5}{*}{$\begin{array}{l}\text { Avaliação da } \\
\text { organização do } \\
\text { processo de } \\
\text { trabalho }\end{array}$} & \\
\hline Reuniões internas com equipe & & \\
\hline Perceber-se como mediador & & \\
\hline A importância de seu papel/responsabilidades & & \\
\hline Humanizar o atendimento & & \\
\hline Descontentamento com a gestão & \multirow{3}{*}{$\begin{array}{c}\text { Organização da } \\
\text { PNAB }\end{array}$} & \\
\hline Responsabilidades da Secretaria Municipal de Saúde & & \\
\hline Falta de programas sociais em saúde para a comunidade & & \\
\hline Cumprimento de metas & \multirow{2}{*}{$\begin{array}{l}\text { Exigências da } \\
\text { Gestão }\end{array}$} & \\
\hline Participação da equipe nas capacitações ofertadas & & \\
\hline
\end{tabular}

Ao serem questionados se conheciam e sobre o que conheciam da PNAB, os entrevistados E3, E5 e E10, apresentaram as seguintes afirmações:

"[...] Sim. Que ela é uma porta de entrada do sistema de... do SUS né, para os usuários, ou seja, os pacientes, população em geral, onde elas são orientadas em relação à seus cuidados né, e à sua saúde". (E3)

"[...] Sim. Então, é... É a participação da comunidade como um todo, as atribuições dos profissionais, o que mais?!... também sei que a PNAB faz parte da política de atenção básica, que é a porta de entrada e do processo de Educação Permanente". (E5)

"[...] Sim. Que ela vai nortear as ações da Atenção Básica, as atribuições de todo mundo, da equipe". (E10)

O conhecimento sobre a PNAB por parte de gestores e dos coordenadores é importante para a consolidação, acompanhamento e avaliação sistemática das ações implementadas, visando à readequação do processo de trabalho, uma vez que a autoavaliação do trabalho é uma atribuição comum prevista a todos os profissionais da $A B$. 
Contudo, ao serem questionados sobre o processo de implementação da PNAB, cerca de $80 \%$ dos entrevistados relataram que este ocorre de maneira vertical, ou seja, hierarquicamente da SEMUS para as UBS através de reuniões mensais.

Nestas reuniões são convocados todos os coordenadores das ESF para discutirem o processo de trabalho das equipes, bem como para avaliação de índices, programas e metas alcançadas. Tal fato é percebido nas falas dos entrevistados E4 e E8.

"[...] É... ocorre da seguinte maneira: vem de cima pra baixo. Nós temos os gestores, que é o nosso coordenador, passa o cronograma das ações e nós enfermeiros realizamos aqui dentro da Unidade junto com a comunidade." (E4)

"[...] Então, geralmente nós temos reuniões mensais na Secretaria Municipal de Saúde com todos os enfermeiros e Coordenadores de Atenção Básica que passam todos os programas, tudo que nós temos que passar por capacitação. Então isso, esse processo de implementação, ele vem da coordenadora de Atenção Básica pros enfermeiros das Unidades Básicas de Saúde em reunião." (E8)

As práticas de saúde desenvolvidas nos serviços do SUS ainda são pautadas na lógica da verticalização de programas, em ações e relações fragmentadas e hierarquizadas entre os profissionais. Embora isso venha sofrendo mudanças pontuais, o processo de construção e consolidação do SUS compõe e impõe pautas nas agendas de discussões das IES brasileiras que oferecem cursos na área da saúde e direcionam os processos de formação de força de trabalho para bem intervir no SUS (LUCCHESE; PEREIRA, 2010).

Por outro lado, o entrevistado $E 11$ foi o único que citou a necessidade da participação de toda a equipe nestas reuniões, porém, em sua fala, o mesmo deixa claro o caráter eventual tanto das reuniões quanto da participação integral da equipe.

"[...] Bom, nós temos capacitações em torno de 3 meses, 4 meses, de 4 em 4 meses, nem sempre é uma data específica, mas sempre temos né, e essas capacitações geralmente o enfermeiro participa, nem sempre é a equipe por inteiro né, aí depois eu repasso o que foi passado pra mim pras demais colegas de trabalho né?! Mas... a princípio é só mesmo o enfermeiro que participa." (E11)

As atribuições dos profissionais das eAB devem seguir as referidas disposições legais que regulamentam o exercício de cada uma das profissões. Portanto, a PNAB apresenta as atribuições comuns a todos os profissionais, e dentre elas destacam-se realizar e participar reuniões de equipes a fim de discutirem em conjunto o planejamento e avaliação das ações da equipe, a partir da utilização dos dados disponíveis e; acompanhar e avaliar sistematicamente as ações implementadas, visando à readequação do processo de trabalho (BRASIL, 2012). 
Além das entrevistas com os coordenadores das ESF, também foi realizado contato com a Coordenação da Atenção Básica da SEMUS para complementar as informações acerca do fluxograma da PNAB conforme Figura 1 abaixo.

Figura 1 - Implementação do Fluxograma da PNAB no município de Gurupi, Tocantins, Brasil.

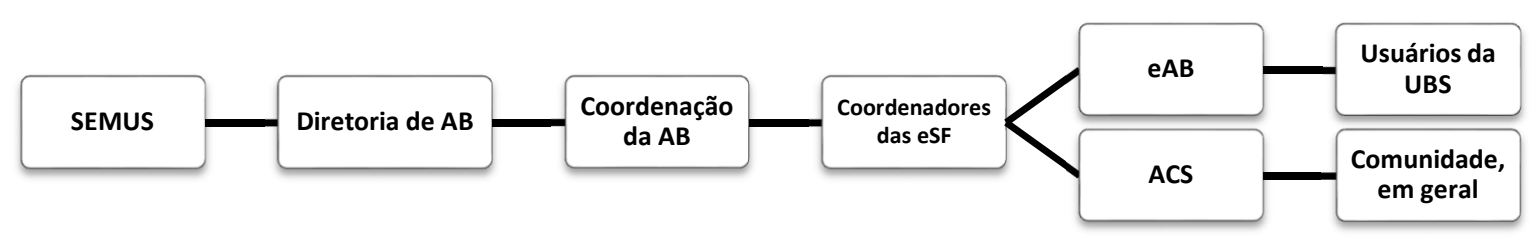

A organização do fluxograma da PNAB no município de Gurupi-TO está caracterizada de modo hierárquico e vertical, na qual centraliza-se na figura do gestor como elemento principal para que todo seu processo aconteça e contemple os usuários e comunidade, em geral.

A valorização da PNAB centraliza-se como esteio para a atuação do gestor em saúde na $A B$, devendo, o mesmo, reconhecer que esta política pública possui todo um histórico de resultados da experiência acumulada por conjunto de atores envolvidos paralelo à consolidação do SUS. Também há de considerar que ela é fruto de lutas e movimentos sociais de usuários, trabalhadores e gestores dos três entes federativos (BRASIL, 2012).

Ao serem questionados se gostariam de acrescentar algo sobre a PNAB, os sujeitos E1 E2 e E5 reconheceram a importância da mesma para toda a equipe profissional, em que o pleno conhecimento sobre informações contidas na mesma facilitaria no processo de trabalho da eSF.

"[...] Eu acho a PNAB muito interessante e eu acho que a gente deveria ter mais acesso, mais conhecimento sobre o que é, sobre o que é as nossas competências sobre ela... Ter mais acesso às informações contidas nela." (E1)

"[...] Eu creio que os profissionais deveriam conhecer mais a respeito dela e também ser mais oferecido isso da coordenação nossa, da nossa gestão, informar mais os profissionais que estão no município. Acontecem mudanças, então sempre que acontecer essas mudanças no município, oferecer uma capacitação, um curso, algo que volte pras pessoas conhecerem a PNAB, que poucos conhecem, ou já ouviu falar ou não tem o conhecimento a fundo do que ela é. Então ela é importante pra mim e pra todos também. Seria importante ter uma divulgação maior." (E2)

“[...] Então, na verdade eu acredito que ainda falta é mais informação, até mesmo para todos os profissionais e mediante a isso, os profissionais do administrativo né, que eles são a porta de entrada aqui da nossa UBS. Então, desde como receber este paciente e até mesmo da função deles que é receber este paciente e dar uma... 
Observa-se que a autoavaliação dos coordenadores sobre o processo de implementação da PNAB nas UBS de Gurupi faz referência ao acesso, divulgação e conhecimento da própria equipe sobre a mesma.

Atualmente, um dos eixos centrais para o pleno aperfeiçoamento e melhoria dos serviços da $A B$ está na autoavaliação. Esta ferramenta teve início em 2005 e passou por reformulação em 2011, assim, iniciou-se uma nova etapa do processo de institucionalização da avaliação da $A B$ no país, com a formulação do Programa Nacional para Melhoria do Acesso e Qualidade da Atenção Básica (PMAQ-AB). Um dos objetivos do PMAQ é institucionalizar a cultura da avaliação (BRASIL, 2011).

O processo de autoavaliação é compreendido como ponto de partida da fase de desenvolvimento do PMAQ, uma vez que os processos orientados para a melhoria da qualidade têm início na identificação e reconhecimento das dimensões positivas e também problemáticas do trabalho da gestão e das equipes de atenção à saúde. Nesse momento, deverão ser produzidos sentidos e significados com potencial de facilitar a mobilização de iniciativas para mudança e aprimoramento dos serviços (BRASIL, 2012).

\section{CONSIDERAÇOES FINAIS}

Esta pesquisa possibilitou conhecer sobre o processo de implementação e organização de políticas públicas de saúde na Atenção Básica. A partir da análise dos relatos dos entrevistados, foi possível retomar à questão norteadoras do estudo e respondêla.

A partir do fluxograma da PNAB, identificou-se seu processo de implementação nas Unidades Básicas de Saúde do município de Gurupi-TO. Assim, percebe-se que o mesmo ocorre verticalmente através de reuniões mensais entre gestores da SEMUS e os enfermeiros coordenadores das equipes, assim, discute-se o processo de trabalho, avaliam-se as metas e planejam ações em saúde para a comunidade.

Compreendeu-se que a cultura da população atendida, as metas a serem alcançadas e as exigências da gestão são fatores que dificultam o processo de implementação da PNAB nas UBS de Gurupi-TO.

Compreender o processo de implementação de uma Política Pública de Saúde no município de Gurupi foi desafiante, contudo, no decorrer das etapas desta pesquisa, foi 
possível conhecer as distintas formas de atuação profissional frente aos desafios da $A B$. Essas reflexões são recortes da realidade político-organizativa local.

Conforme relato da gestão local, o departamento de Direção e Coordenação de Atenção Básica do Município de Saúde de Gurupi, responsável pela gestão e coordenação das UBS, está disposto a receber propostas, projetos e sugestões que objetivem colaborar com a implementação e organização de políticas públicas de saúde locais.

Não se pretende esgotar a discussão a respeito do tema em questão. Sugere-se a realização de estudos que aprofundem a compreensão e reflexão acerca da implementação de políticas públicas de saúde na $A B$ e que desencadeiem transformações no cotidiano dos serviços de saúde. Sabe-se que o modelo de gestão implementado ainda carrega consigo aspectos que rompem com os objetivos do modelo assistencial instituído.

\section{REFERÉNCIAS}

BARDIN, Laurence. Análise de conteúdo. 4ª ed. Lisboa: Edições; 2009.

BRASIL. Constituição (1988). Constituição da República Federativa do Brasil. Brasília, DF: Senado Federal: Centro Gráfico, p. 292, 1988. Disponível em: http://www.planalto.gov.br/ccivil 03/constituicao/constituicao.htm. Aceso em 15 abr 2017.

Ministério da Saúde. Portaria GM/MS n. 1.996, de 20 de agosto de 2007. Dispõe sobre as diretrizes para a implementação da Política Nacional de Educação Permanente em Saúde. Brasília, 2009. Disponível em: http://bvsms.saude.gov.br/bvs/publicacoes/politica_nacional_educacao_permanente_saud e.pdf. Acesso em 12 jul 2016.

- Ministério da Saúde. Secretaria de Atenção à Saúde. Política Nacional de Humanização da Atenção e Gestão do SUS. Humaniza SUS: clínica ampliada e compartilhada. Brasília, DF, 2009. Disponível em: http://bvsms.saude.gov.br/bvs/publicacoes/humaniza sus atencao basica.pdf. Acesso em 12 mai 2017.

. Ministério da Saúde. Secretaria de Atenção à Saúde. Departamento de Atenção Básica. Programa Nacional de Melhoria do Acesso e da Qualidade da Atenção Básica. Portaria 1.654, de 19 de julho de 2011. Brasília: Ministério da Saúde, 2011. Disponível em: http://189.28.128.100/dab/docs/portaldab/documentos/pmaq/prt_1645_02_10_2015.pdf. Acesso em 13 abr 2017.

. Conselho Nacional de Saúde. Resolução n. 466, de 12 de dezembro de 2012, que trata de pesquisas em seres humanos. Brasília: CNS; 2012. Disponível em: http://conselho.saude.gov.br/resolucoes/2012/Reso466.pdf. Acesso em 06 Jun 2015.

. Ministério da Saúde. Secretaria de Atenção à Saúde. Departamento de Atenção Básica. Política Nacional de Atenção Básica (PNAB). Brasília, 2012. Disponível em: http://bvsms.saude.gov.br/bvs/publicacoes/politica_nacional_atencao_basica.pdf. Acesso em 15 mai de 2016 
DESLAURIERS, J.P; KÉRISIT, M. O delineamento de pesquisa qualitativa. In: A pesquisa qualitativa: enfoques epistemológicos e metodológicos. Tradução de Ana Cristina Nasser. Petrópolis, RJ: Vozes. p. 127-153, 2008.

GIL, Antonio Carlos. Como elaborar projetos de pesquisa. 4를 ed. São Paulo: Atlas, 2008.

GIOVANELLA, Ligia et al. Saúde da família: limites e possibilidades para uma abordagem integral de atenção primária à saúde no Brasil. Ciênc saúde coletiva, v. 14, n. 3, p. 783-94, $2009 . \quad$ Disponível em: $<\mathrm{http} / / / \mathrm{www}$.institutoconscienciago.com.br/pdf/saude/limites_possibilidades.pdf> acesso em 21 mar 2017.

GUERRA, Eliane Linhares de Assis. Manual de Pesquisa Qualitativa. Anima Educação. Belo Horizonte, 2014.

LUCCHESE, Roselma; VERA, Ivânia; PEREIRA, Wilza Rocha. As políticas públicas de saúde-SUS-como referência para o processo ensino-aprendizagem do enfermeiro. Revista Eletrônica de Enfermagem, v. 12, n. 3, p. 562-6, 2010. Disponível em: https://www.revistas.ufg.br/fen/article/view/11144. Acesso em 14 abr 2017.

MINAYO, Maria Cecília de Souza. O desafio do conhecimento: pesquisa qualitativa em saúde. São Paulo: Hucitec, 2007.

SOUZA, Renata Odete de Azevedo; MACHADO, Cristiani Vieira; NORONHA, Marina Ferreira de. Desafios da Gestão Municipal de Atenção Básica em Saúde no Brasil: Um estudo de caso. Rev. APS, v. 18, n. 2, 2015. Disponível em: https://aps.ufjf.emnuvens.com.br/aps/article/view/2476. Acesso em 07 abr 2017.

SUNDFELD, Ana Cristina. Clínica ampliada na atenção básica e processos de subjetivação: relato de uma experiência. Physis, v. 20, n. 4, p. 1079-1097, 2010. Disponível em: http://www.scielosp.org/scielo.php?script=sci_arttext\&pid=S0103$73312010000400002 \&$ Ing=pt\&nrm=iso. Acesso em 11 mai 2017. 